\title{
Covid Media Misguidance: A Case Report Highlighting the Influence of Media on Patient Medication Decision Making
}

Shaylee Anderson, Pharm.D Candidate ${ }^{1,2}$; Joe Windscheffel, PharmD, BCACP ${ }^{1,2,3}$; Karen Gunning, PharmD, BCPS, BCACP, FCCP ${ }^{1,2,3}$

${ }^{1}$ University of Utah College of Pharmacy; ${ }^{2}$ University of Utah Health; ${ }^{3}$ University of Utah Department of Family \& Preventive Medicine

\begin{abstract}
Purpose: This case report details the influence of media on patients and the responsibility of health care providers to educate their patients on proper use of medications, and to be aware of potential misadventures based on messages in popular media.

Summary: The sudden rise of the COVID19 pandemic has led to media outlets reporting science without necessary peer review and has resulted in preliminary data presented as factual evidence. It is difficult for patients without an extensive medical background in science to fully understand the uncertainty of information shared in popular media. This was demonstrated when preliminary data showed potential promise of hydroxychloroquine for the treatment/prevention of COVID19. This led to patients requesting hydroxychloroquine prescriptions from their providers, as well as stockpiling medication, which led to a shortage. In addition, patients began taking chloroquine containing substances not intended for human consumption. Popular media created a belief in the general public that all antimalarial drugs may work to prevent COVID19. This case report presents an elderly patient that presented to clinic with shortness of breath and lightheadedness. Upon interviewing the patient, it was discovered that he had been taking an old supply of atovaquone and proguanil hydrochloride. Physical exam, and laboratory examination were evaluated to rule out any other etiology with all tests and exams being unremarkable. Two weeks after stopping atovaquone and proguanil hydrochloride, the patient's symptoms completely resolved.

Conclusion: The media provides a significant portion of the information that patients receive regarding rapidly changing treatment information in a pandemic. It is crucial for health care providers to know what information patients are exposed to, and to educate patients with evidence-based information. Pharmacists are the most accessible health care providers and have a key role in medication review and management. Educating patients on evidence-based use of medications may help avoid harm caused by misinformation from unreliable media sources.
\end{abstract}

Keywords: COVID19, atovaquone-proguanil hydrochloride, pharmacist, media, antimalarial

\section{Introduction}

With the rise of the COVID19 (SARS-CoV-2) pandemic, studies of antimalarial drugs have reemerged for the treatment and/or prevention of coronaviruses. After the outbreak of Middle East Respiratory Syndrome (MERS) in 2012, a random screen of medications was conducted in which chloroquine was shown to inhibit coronaviruses in vivo. ${ }^{1}$ There is not a clear understanding of how chloroquine acts against coronavirus, however there are a few proposed mechanisms of action. Chloroquine is used to treat malaria with significant risk of toxicity. Hydroxychloroquine is a less toxic metabolite of chloroquine, more commonly used to treat rheumatic disease. ${ }^{2}$ Since chloroquine showed promise in vivo against previous coronaviruses (MERS), researchers were hopeful that hydroxychloroquine, being less toxic, would have a similar effect on SARS-CoV-2. ${ }^{1}$

Corresponding author: Shaylee Anderson, PharmD Candidate University of Utah College of Pharmacy

University of Utah Health

Email: shaylee.anderson@pharm.utah.edu
A study published in late March 2020 examined 20 patients treated with $600 \mathrm{mg}$ of hydroxychloroquine daily, and showed a reduction in the viral load and lower average carrying duration than those untreated. ${ }^{3}$ After this study was published, President Trump publicized the use of hydroxychloroquine and other antimalarial drugs as "game changers", and later revealed he was taking hydroxychloroquine for the prevention of COVID19. ${ }^{4}$ After this press conference there was a national rush to obtain hydroxychloroquine that led to a shortage. ${ }^{1,5,6}$ The week directly after President Trump's endorsement, hydroxychloroquine prescriptions increased nearly $200 \%$ with 483,425 excess fills over a 10-week period compared to 2019 . $^{7}$ A study published in JAMA sought to quantify changes in chloroquine and azithromycin prescriptions from 10/2019$3 / 2020 .^{8}$ They found in 3/2020 compared to $10 / 2019-2 / 2020$ the number of chloroquine prescriptions increased $158.6 \%$, hydroxychloroquine increased $86.2 \%$, and the number of patients receiving both hydroxychloroquine and azithromycin increased $1044 \%{ }^{8}$ As a result of the media focus on hydroxychloroquine as a promising treatment, misinformation facilitated the utilization of antimalarial medications for the prevention/treatment of COVID19. ${ }^{7}$ 
Malarone (atovaquone and proguanil hydrochloride), an antimalarial medication, has not been studied or connected to the prevention or treatment of COVID19. This medication can cause several side effects including dizziness (5\%) and asthenia (8\%). Post marketing adverse events reported include anemia, immune system disorders, and hepatobiliary disorders. ${ }^{9}$ Although this medication has no scientific evidence supporting its use for COVID19, patients have been exposed to sources stating "antimalarial drugs" will provide them protection from COVID19 which led to many taking any antimalarial medications or chloroquine containing substances that can lead to adverse effects, hospitalization and even death. ${ }^{1}$ Media has the potential to influence the public to self-medicate without the direction or with minimal direction from medical providers.

\section{Case Presentation}

A 78 year old male, presented to their primary care physician with shortness of breath (SOB) and light-headedness. The patient reported no symptoms during physical activity but did become symptomatic at rest. The patient stated they actively monitored oxygen saturation, heart rate, and blood pressure during these bouts of SOB and light-headedness, all of which were within normal limits. Lab results from visit are available in table 1 , all lab results and vital sign were within normal limits. An electrocardiogram and chest $x$-ray were not completed due to normal physical exam findings. The patient had a remaining supply of atovaquone/proguanil hydrochloride $250 \mathrm{mg} / 100 \mathrm{mg}$ (expiration: 1/31/21) from previous international travel, four years prior to presentation. The patient reported no adverse effects from this medication while taking it for malaria prevention. The patient explained that one of their children had heard from popular media that antimalarial medications could be used to treat/prevent COVID19, so out of concern for personal health, the patient began taking the atovaquone/ proguanil hydrochloride daily 6 weeks prior to presentation to their primary care provider. Symptoms started, unprovoked, about 5-6 weeks prior to visit. At the time of visit the patient's current medication regimen was as follows: ipratropium $0.6 \% 2$ sprays in each nostril daily, vitamin b12 daily, vitamin d3 daily, multiple vitamin daily, vitamin e daily, fish oil daily, and folic acid daily. The patient has no medication allergies. Past medical history was not pertinent to the current complaints. At the visit, the patient was instructed to discontinue the atovaquone/proguanil hydrochloride. A COVID19 test was performed (negative), a complete metabolic panel, complete blood count, b-type natriuretic peptide, and ferritin were drawn that were all either within normal limits or unchanged from patient's baseline labs. Two weeks after stopping atovaquone/proguanil hydrochloride the patient reported the SOB and light-headedness had completely resolved. The etiology of the patient's symptoms in unknown, but given the timeline of their appearance and disappearance, it is reasonable to assume atovaquone/proguanil hydrochloride may have been a contributing factor. A Naranjo nomogram was calculated at 6, indicating atovaquone/proguanil hydrochloride was the probable cause of this patient's symptoms. ${ }^{12}$

\section{Discussion}

The American public is constantly exposed to information through social media and unsubstantiated news sources. Many assume the information being shared from these sources is credible and unfortunately take this information as factual. Media sources are not required to screen information for accuracy before releasing it to the public. The reporting of this type of information has the potential to cause great harm to the public. This was seen when preliminary evidence about the use of hydroxychloroquine for the treatment/prevention of the novel SARS-CoV-2 was shared with the public. The sharing of this information, along with President Donald Trump's endorsement led to a jump in use, resulting in a shortage of hydroxychloroquine. Patients that rely on this medication for lupus and rheumatoid arthritis began having difficulty obtaining their medication. 5,10 Due to this shortage, the Federal Drug Administration (FDA) released a statement that all manufactures were ramping up production to ensure these patients could receive their medication and studies could still be conducted. ${ }^{6}$ Along with the FDA statement, many major medical organizations released statements that emphasized rational use of hydroxychloroquine in patients with COVID19, such as in studies, until reliable evidence is available. ${ }^{6,10}$ Along with the shortage of hydroxychloroquine there were reports of patients using atovaquone/proguanil for COVID19 prophylaxis presenting with QT prolongation. ${ }^{2}$ There was also a case report from Arizona about a couple that used a pesticide containing chloroquine for the prevention of COVID19 that led to the death of one and the hospitalization of the other. ${ }^{1}$

In this particular case, the patient had heard from his family only a portion of information that "antimalarial drugs may prevent COVID19". He accepted this information as fact, and assumed all antimalarial medications had the potential to prevent COVID19. Without question or under direction of a health care provide, he began taking atovaquone/proguanil hydrochloride. Fortunately, this patient only experienced mild side effects from taking a medication for an unindicated purpose, and had access to a primary care provider and clinical pharmacy team that collected comprehensive information that caused them to instruct him to stop the unnecessary medication.

\section{Conclusion}

Information regarding proposed medical treatments has the ability to spread faster than ever before, which allows conspiracy theories and unproven statements to reach a large population. The constitutional right to freedom of speech limits the ability to legally prevent the spread of false information. Laws set in place in other countries have received pushback due to government regulation of what is "true" and what is not. While intentions in other countries were to stop the spread of unsubstantiated information, this ruling led to an unrest in society. ${ }^{11}$ This has proven to be a difficult problem for many government officials and scientists during the COVID19 outbreak. There is a positive aspect to the instantaneous access 
to information as seen with implementation of social distancing and quarantine measures. ${ }^{13}$ Due to the vast audience that social platforms have, there is an increasing responsibility for those on news stations and social media to ensure the information they share is safe, rational, and evidence-based. There is also an increased responsibility for health care providers to understand what information their patients may be presented with and how to help their patients navigate the vast amount of "medical" information they receive daily. This is an excellent opportunity for pharmacists to educate their patients on evidence-based use of all medications and dietary supplements.

It is critical for health care providers to understand the impact media has on their patients. It is also important to know what information their patients are receiving and to educate them with evidence-based information. Pharmacists can play a large role in helping patients understand the risks of their mediations and the importance of only taking medications for indicated purposes. Pharmacists are the most accessible members of the health care community for patients, giving them the opportunity to screen and educate patients on the correct use of their medications, and to answer questions about medications that may be promoted via the media.

Conflict of Interest: We declare no conflicts of interest or financial interests that the authors or members of their immediate families have in any product or service discussed in the manuscript, including grants (pending or received), employment, gifts, stock holdings or options, honoraria, consultancies, expert testimony, patents and royalties.

Treatment of Human Subjects: none

Table 1: Lab results from first presentation to PCP workup.

\begin{tabular}{|c|c|}
\hline Test & Result \\
\hline Vitals & $130 / 85 \mathrm{mmHg}$ \\
Blood Pressure & $70 \mathrm{BPM}$ \\
Heart Rate & $97 \%$ \\
Oxygen Saturation & Negative \\
\hline COVID19 & $81 \mathrm{pg} / \mathrm{mL}$ \\
\hline B-Type Natriuretic & $186 \mathrm{ng} / \mathrm{mL}$ \\
Peptide & WBC $(4.28 \mathrm{k} / \mathrm{uL})$ \\
\hline Ferritin & $\mathrm{RBC}(4.39 \mathrm{M} / \mathrm{uL})$ \\
\hline CBC & $\mathrm{HGB}(14.0 \mathrm{~g} / \mathrm{dL})$ \\
*Results consistent with & $\mathrm{HCT}(41.4 \%)$ \\
\hline previous CBC* & Unremarkable \\
\hline BMP & Unremarkable \\
\hline CMP & $83 \mathrm{~mL} / \mathrm{min} / 1.73 \mathrm{BSA}$ \\
\hline eGFR &
\end{tabular}

\section{References}

1. Seley-Radtke K. What's The Deal With Antimalarials And Coronavirus? Read This Before You Try Anything. Science Direct. 2020. https://www.sciencealert.com/when-it-comes-to-treatingcovid-19-chloroquine-is-problematic-and-unproven. Accessed June 16, 2020.

2. Stokkermans TJ. Chloroquine And Hydroxychloroquine Toxicity. StatPearls [Internet].

https://www.ncbi.nlm.nih.gov/books/NBK537086/. Published May 29, 2020. Accessed June 16, 2020.

3. Lover AA. Quantifying treatment effects of hydroxychloroquine and azithromycin for COVID-19: a secondary analysis of an open label non-randomized clinical trial (Gautret et al, 2020). International Journal of Antimicrobial Agents. April 2020. doi:10.1101/2020.03.22.20040949.

4. DeJong C, Wachter RM. The Risks of Prescribing Hydroxychloroquine for Treatment of COVID-19-First, Do No Harm. JAMA Intern Med. Published online April 29, 2020. doi:10.1001/jamainternmed.2020.1853

5. Lupus Foundation. FDA Recognizes Hydroxychloroquine and Chloroquine Shortages. Lupus Foundation of America. https://www.lupus.org/news/fda-recognizes-hydroxychloroquineand-chloroquine-shortages. Published 2020. Accessed June 16, 2020.

6. Center for Drug Evaluation and Research. FDA cautions use of hydroxychloroquine/chloroquine for COVID-19. U.S. Food and Drug Administration. https://www.fda.gov/drugs/drug-safety-andavailability/fda-cautions-against-use-hydroxychloroquine-orchloroquine-covid-19-outside-hospital-setting-or. Published 2020. Accessed June 23, 2020.

7. Vaduganathan M, van Meijgaard J, Mehra MR, Joseph J, O'Donnell CJ, Warraich HJ. Prescription Fill Patterns for Commonly Used Drugs During the COVID-19 Pandemic in the United States. JAMA. 2020;323(24):2524-2526. doi:10.1001/jama.2020.9184

8. Shehab, N., Lovegrove, M., \& Budnitz, D. S. (2020). US Hydroxychloroquine, Chloroquine, and Azithromycin Outpatient Prescription Trends, October 2019 Through March 2020. JAMA Internal Medicine, 180(10), 1384-1386.

9. GalaxoSmithKline. Highlights of prescribing information: Malarone. gsksourcecom. 2000.

https://www.gsksource.com/pharma/content/dam/GlaxoSmithKlin e/US/en/Prescribing_Information/Malarone/pdf/MALARONE.PDF. Accessed June 16, 2020.

10. Kim AHJ, Sparks JA, Liew JW, et al. A Rush to Judgment? Rapid Reporting and Dissemination of Results and Its Consequences Regarding the Use of Hydroxychloroquine for COVID-19 [published correction appears in Ann Intern Med. 2020 Jun 16;172(12):844]. Ann Intern Med. 2020;172(12):819-821. doi:10.7326/M20-1223

11. Kirtley JE. Getting to the Truth: Fake News, Libel Laws, and "Enemies of the American People". American Bar Association. https://www.americanbar.org/groups/crsj/publications/human_rig hts_magazine_home/the-ongoing-challenge-to-define-freespeech/getting-to-the-truth/. Published October 20, 2018. Accessed June 23, 2020.

12. Naranjo algorithm: A method for estimating the probability of adverse drug reactions. Pmidcalc.org. Accessed December 2, 2020. http://www.pmidcalc.org/?sid=7249508\&newtest $=Y$

13. Ali SH, Kurasawa F. \#COVID19: Social media both a blessing and a curse during coronavirus pandemic. The Conversation. https://theconversation.com/covid19-social-media-both-ablessing-and-a-curse-during-coronavirus-pandemic-133596. Published May 7, 2020. Accessed June 16, 2020. 